\title{
Electron transport through silicon serial triple quantum dots
}

\author{
Gento Yamahata $^{\mathrm{a}, *}$, Yoshishige Tsuchiya ${ }^{\text {b,c }}$, Hiroshi Mizuta ${ }^{\text {b,c,d }}$, Ken Uchida ${ }^{\text {c,d }}$, Shunri Oda ${ }^{\text {a,c }}$ \\ ${ }^{a}$ Quantum Nanoelectronics Research Center, Tokyo Institute of Technology, 2-12-1 O-okayama, Meguro-ku, Tokyo 152-8552, Japan \\ ${ }^{\mathrm{b}}$ School of Electronics and Computer Science, University of Southampton, Highfield, Southampton SO17 1BJ, United Kingdom \\ ' SORST-JST, Chiyoda-ku, Tokyo 102-0075, Japan \\ ${ }^{\mathrm{d}}$ Department of Physical Electronics, Tokyo Institute of Technology, 2-12-1 O-okayama, Meguro-ku, Tokyo 152-8552, Japan
}

\section{A R T I C L E I N F O}

\section{Article history:}

Received 10 November 2008

Accepted 9 February 2009

Available online 24 April 2009

The review of this paper was arranged by Prof. P. Ashburn

\section{Keywords:}

Silicon

Coulomb blockade

Triple quantum dots

\begin{abstract}
A B S T R A C T
We study the electron transport through silicon serial triple quantum dots (TQDs) formed effectively in a lithographically-defined multiple quantum dot system on a silicon-on-insulator substrate at a temperature of $4.2 \mathrm{~K}$. Our serial TQDs are composed of two lithographically-patterned QDs and another one inbetween formed by stress during the pattern-dependent oxidation process. The TQDs formation is confirmed by equivalent circuit simulations, which show an excellent agreement with the experimental results. With detailed analysis of the charge configurations in the TQDs, we discuss the distinct properties of the TQDs, including electron transport at the charge quadruple points. In addition, we discuss higher order tunneling processes of the TQDs. The analysis of electron states in the silicon TQDs is a crucial step toward the future implementation of integrated silicon quantum information devices.
\end{abstract}

(c) 2009 Elsevier Ltd. All rights reserved.

\section{Introduction}

Silicon double quantum dots (DQDs) are promising candidates for developing quantum information devices based on electronic state or electron spin quantum bits (qubits). So far, coherent manipulation of the qubits has been demonstrated mainly by DQDs in GaAs systems [1-3]. However, several deleterious effects, such as the hyperfine interaction, the spin-orbit interaction, and the piezoelectric electron-phonon interaction, severely limit coherence times [1-4]. Longer coherence times are expected in silicon DQDs because of the predominantly spin-zero nuclei, weak spin-orbit coupling, and the lack of piezoelectric electron-phonon coupling [5]. In addition, silicon qubits have a great advantage in integration because of the compatibility with conventional silicon complementary metal-oxide-semiconductor processes.

In order to investigate electron transport through silicon DQDs, several experimental studies have been carried out, including studies on gate-defined DQDs [6,7], ion implanted Si:P DQDs [8], and $\mathrm{Ge} / \mathrm{Si}$ core/shell nanowires [9]. Furthermore, the spin dependent electron transport referred to as spin blockade due to the Pauli exclusion principle in the tunneling characteristics $[10,11]$ has been demonstrated in DQDs in Si/Ge two-dimensional electron gases [12] and in naturally-formed DQDs in silicon nanowires [13]. This phenomenon is a valuable tool for the measurement and manipulation of individual electron spins [2,3]. Additionally,

\footnotetext{
* Corresponding author.

E-mail address: ygent@neo.pe.titech.ac.jp (G. Yamahata).
}

the multivalley conduction band structures of silicon in DQDs [14] have been highlighted for further investigation.

A triple quantum dot (TQD) system is the natural extension of the DQDs toward scalable multiqubit systems. Theoretical studies of TQDs indicate strong potential for applications in quantum information technology. To improve the coherence time, coded qubits with TQDs have been studied $[15,16]$. Spin cluster qubits, where no local control of spins is required, have also been proposed with a TQD system as the minimum system [17]. Furthermore, entangled electron spins are expected to be created by TQDs as spin-entangled currents [18]. In addition to applications in quantum information processing, charging rectifiers using TQDs have been proposed and demonstrated $[19,20]$. Recently, experimental electron transport of TQDs has been investigated in AlGaAs/GaAs heterostructures [21-23], a single-wall carbon nanotube [24], and self-assembled InAs quantum dots [25]. However, clear electron transport characteristics for silicon TQDs have not been reported yet.

In this paper, we demonstrate electron transport through silicon serial TQDs formed effectively in a lithographically-defined multiple quantum dot (QD) system which has several strong constrictions. Similar configurations have been studied as the silicon-on-insulator QCA cell $[26,27]$. The TQDs include a very small quantum dot arising from the compressive stress during the thermal oxidation process. The equivalent circuit simulation supports the claim of the formation of the TQD system. In Section 2, we introduce the device structure and the fabrication processes. Section 3 is devoted to discussing the validity of the assumed QD 


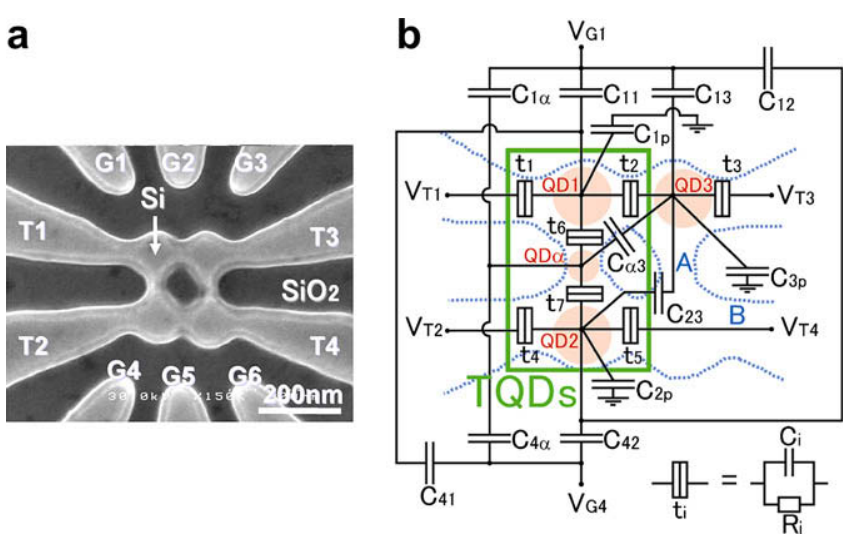

Fig. 1. (a) Scanning electron microscope (SEM) image of the silicon multiple quantum dot (QD) system. The bright (dark) region consists of silicon (silicon dioxide). T1, T2, T3, and T4 are the terminals connected to the multiple QDs, and G1, G2, G3, G4, G5, and G6 are the side gates. (b) The equivalent circuit of the multiple OD system with the capacitors $C$, the voltage sources $V$, and the tunnel junctions $t$. The tunnel junctions $t_{i}(i=1 \cdots 7)$ are modeled as capacitors $C_{i}$ and resistors $R_{i}$ connected in parallel as shown in the bottom right region. The red circles indicate the effectively formed QDs (QD1, QD2, QD3, and QD $\alpha$ ). The two gates, G1 and G4, which are used in the measurement are included in the circuit. $C_{1 p}, C_{2 p}$, and $C_{3 p}$ are parasitic capacitances, which arise from the couplings between QDs and other electrodes neglected in the circuit. The parasitic capacitance for $\mathrm{QD} \alpha$ can be neglected because QD $\alpha$ is screened with QD1 and QD2.

formation, by analyzing the electron transport characteristics. In Section 4, we show a detailed analysis of electron transport through the TQD system with calculated charge configurations.

\section{Device structure and fabrication process}

We fabricated a lithographically-defined silicon multiple QD system, in which TQDs are effectively formed. Fig. 1a shows the scanning electron microscope (SEM) image of the silicon multiple QD system, which has the eight constrictions, defined on a silicon-on-insulator (SOI) substrate with a silicon layer thickness of about $20 \mathrm{~nm}$ on a $200-\mathrm{nm}$-thick buried oxide layer. The multiple QDs are connected to the four terminals (T1, T2, T3, and T4), where bias voltages are applied. The electrochemical potentials in the QDs can be modulated with six side gates (G1, G2, G3, G4, G5, and G6).

First, the 40-nm-thick SOI film, whose thickness was reduced via thermal oxidation, was heavily doped by ion implantation ( $\mathrm{n}$ type, phosphorous, doping concentration $\sim 1 \times 10^{19} \mathrm{~cm}^{-3}$ ). Multiple QDs were then patterned using high-resolution electron beam lithography with the positive resist ZEP520A. Electron cyclotron resonance reactive ion etching was used to transfer the resist pattern into the SOI layer with $\mathrm{CF}_{4}$ used as the etching gas. Thermal oxidation was then performed for $30 \mathrm{~min}$ at $1000^{\circ} \mathrm{C}$ in order to passivate the surface states and reduce the size of the QDs. The thickness of the SOI layer was also reduced to about $20 \mathrm{~nm}$ during this process. Finally, 300-nm-thick Al electrodes were formed by evaporation.

\section{Discussion for the formed QDs}

Fig. 1b shows the effectively formed QDs in the multiple QD system with the equivalent circuit, where the dotted blue ${ }^{1}$ lines indicate the outer shape of the multiple QD system. From the detailed analysis of the experimental and simulated results discussed below, it was concluded that the four QDs (QD1, QD2, QD3, and QD $\alpha$ ) illustrated by the four red circles in Fig. 1b were formed effectively with

\footnotetext{
${ }^{1}$ For interpretation of color in Figs. 1-6, the reader is referred to the web version of
} this article. the seven tunnel junctions $t_{i}(i=1-7)$. In this paper, we focus on electron transport through the serial TQDs formed on the left side of the multiple QDs as indicated by the green square in Fig. $1 \mathrm{~b}$.

All measurements were performed using the Agilent 4156A parameter analyzer at a temperature of $4.2 \mathrm{~K}$ in liquid helium. Since capacitive coupling between the gate G1(G4) and QD1(QD2) is expected to be strong, the voltages applied to G1 and G4 $\left(V_{\mathrm{G} 1}\right.$ and $\left.V_{\mathrm{G} 4}\right)$ were used to efficiently control the electrochemical potentials in the TQDs. Fig. 2a-d shows the contour plots of the currents $I_{\mathrm{T} 1}, I_{\mathrm{T} 2}, I_{\mathrm{T} 3}$, and $I_{\mathrm{T} 4}$ measured at the terminals T1, T2, T3, and $\mathrm{T} 4$, respectively, as a function of the gate voltages $V_{\mathrm{G} 1}$ and $V_{\mathrm{G} 4}$, where the voltages applied to $\mathrm{T} 1, \mathrm{~T} 2$, and $\mathrm{T} 3$ $\left(V_{\mathrm{T} 1}, V_{\mathrm{T} 2}\right.$, and $\left.V_{\mathrm{T} 3}\right)$ are $-6 \mathrm{mV}$ and the voltage to $\mathrm{T} 4\left(V_{\mathrm{T} 4}\right)$ is $0 \mathrm{mV}$. The electron configurations of the multiple QDs strongly influence these characteristics, which are referred to as charge stability diagrams [29].

Then we simulated these properties using a Monte Carlo technique with the equivalent circuit (Fig. 1b). We carefully extracted the gate capacitances from the experimental results and optimized the tunnel junction parameters with more than 200 iterations. The simulated charge stability diagrams as shown in Fig. 2e-f, corresponding to the experimental results in Fig. 2a-d, respectively, show an excellent agreement with the experimental results. In particular, the charge configurations are almost perfectly reproduced. Therefore, the simulations support the assumed QDs formation. In addition, since $V_{\mathrm{T} 1}, V_{\mathrm{T} 2}$, and $V_{\mathrm{T} 3}$ have the same potentials and the current paths are from $\mathrm{T} 1, \mathrm{~T} 2$, and $\mathrm{T} 3$ to $\mathrm{T} 4$, the current peaks of these charge stability diagrams, particularly $I_{\mathrm{T} 1}$, strongly reflect the electron configurations of the serial TQDs (QD1, QD2, and $\mathrm{QD} \alpha$ ). To the best of our knowledge, this is the first demonstration of electron transport through silicon serial TQDs.

We will now discuss the validity of the absence of the right side tunnel junction as indicated by A in Fig. 1b. Fig. 3a shows the electrical characteristics of $I_{\mathrm{T} 1}, I_{\mathrm{T} 2}, I_{\mathrm{T} 3}$, and $I_{\mathrm{T} 4}$ as a function of $V_{\mathrm{T} 1}$, where $V_{\mathrm{T} 2}, V_{\mathrm{T} 3}$, and $V_{\mathrm{T} 4}$ are $0 \mathrm{mV}$. In the low biased region, which is the inside of the two red dotted lines, only $I_{\mathrm{T} 1}$ and $I_{\mathrm{T} 3}$ flow, indicating the electron transport only through QD1 and QD3. In the high biased region, $I_{\mathrm{T} 2}$ and $I_{\mathrm{T} 4}$ start to flow indicated by red ovals in Fig. 3a because Coulomb blockade among QD1, QD $\alpha$, and QD2 is lifted. However, when we apply just the voltage $V_{\mathrm{T} 3}$ as shown in Fig. 3b, only $I_{\mathrm{T} 1}$ and $I_{\mathrm{T} 3}$ are detected. In this case, the conduction path is just QD1 and QD3. This is attributed to the weak or almost disconnected right side interconnection. Additionally, in Fig. 2a and $\mathrm{c}$, the current peaks of $I_{\mathrm{T} 3}$ are very weak compared to those of $I_{\mathrm{T} 1}$, in spite of the same bias voltages being applied to T1 and T3. As the connection A is absent, the four QDs exist in the conduction path from T3 to T4, resulting in the low current amplitude.

Next, the connection B indicated in Fig. 1b is discussed. At the lower left region of Fig. $2 \mathrm{~b}\left(I_{\mathrm{T} 2}\right)$, the electron configurations of the TQDs have a strong influence on the current peaks, whereas parallel current peak lines are observed at the upper right region of Fig. $2 b$, in which the currents through the TQDs are blocked [see Fig. 2a], indicating that a single QD is responsible for the current oscillation. In addition, $I_{\mathrm{T} 2}$ and $I_{\mathrm{T} 4}$ have almost the same current levels in Fig. 3a. If there had been a QD between $t_{5}$ and $\mathrm{B}$ in Fig. $1 \mathrm{~b}, I_{\mathrm{T} 2}$ would be different from $I_{\mathrm{T} 4}$. Therefore, only QD2 is formed in the left bottom region of the multiple QD system.

Here, we discuss the possible formation mechanism of the effectively formed four QDs. Since the bottom of the conduction bands rises due to the quantum-mechanical size effects in the lithographically-defined strong constrictions, the seven tunnel barriers are formed. However, at the two constrictions indicated by A and $B$ in Fig. 1b, the tunnel junctions are not effective as discussed previously; the coupling at the constriction $A$ is weak because of the very strong constriction, whereas the coupling at the constriction $B$ is strong because of the very weak constriction. In the re- 
a

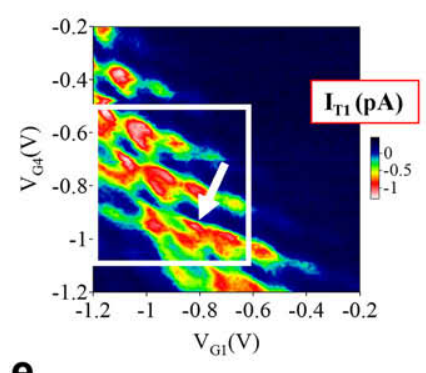

e

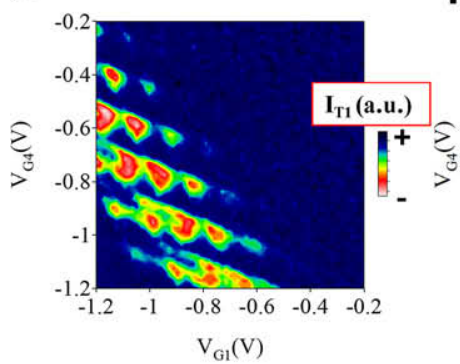

b

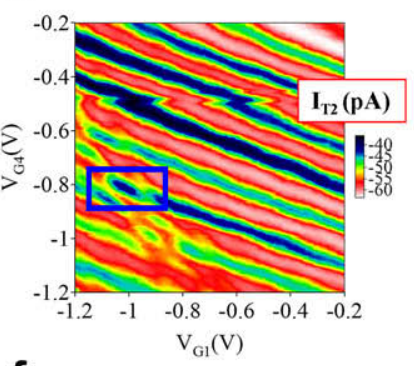

f

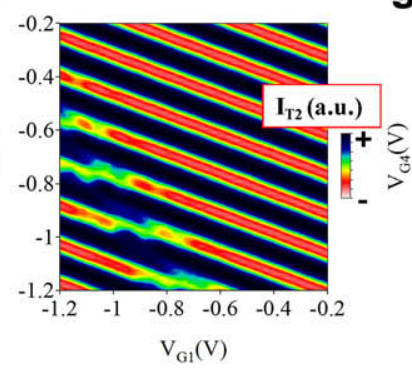

C

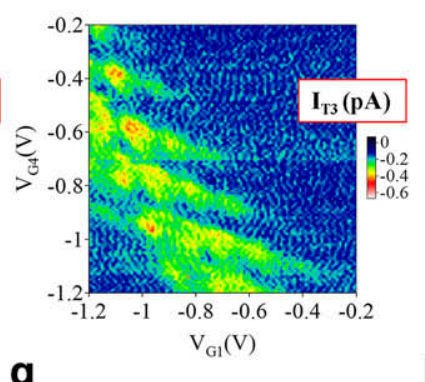

g

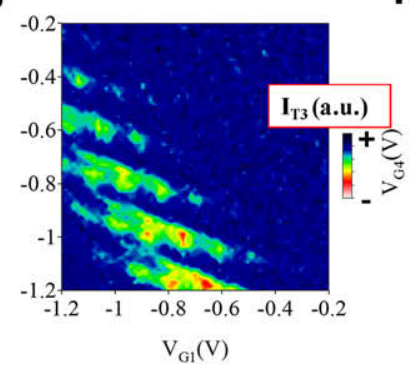

d

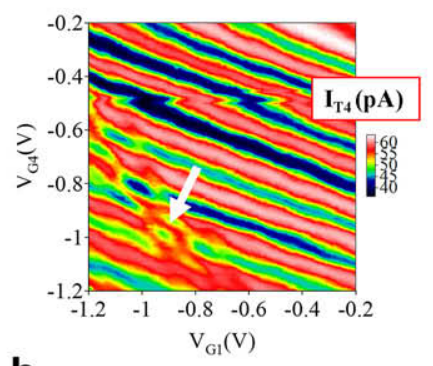

h

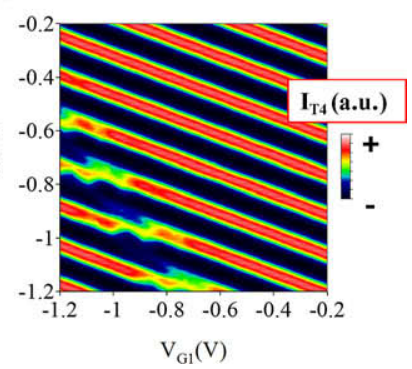

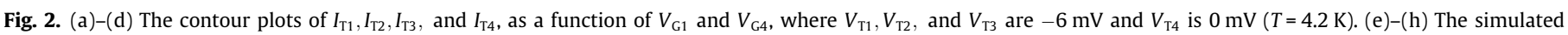

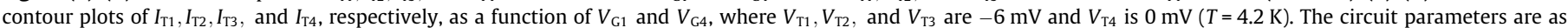

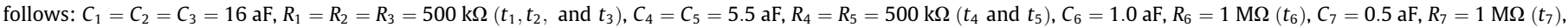

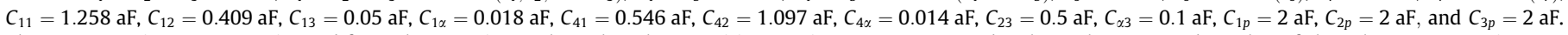

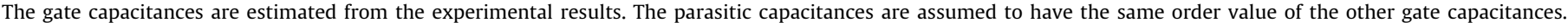

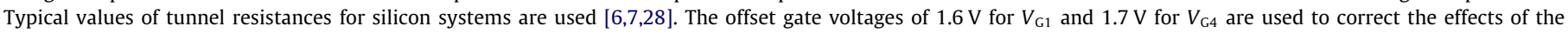
background charges.
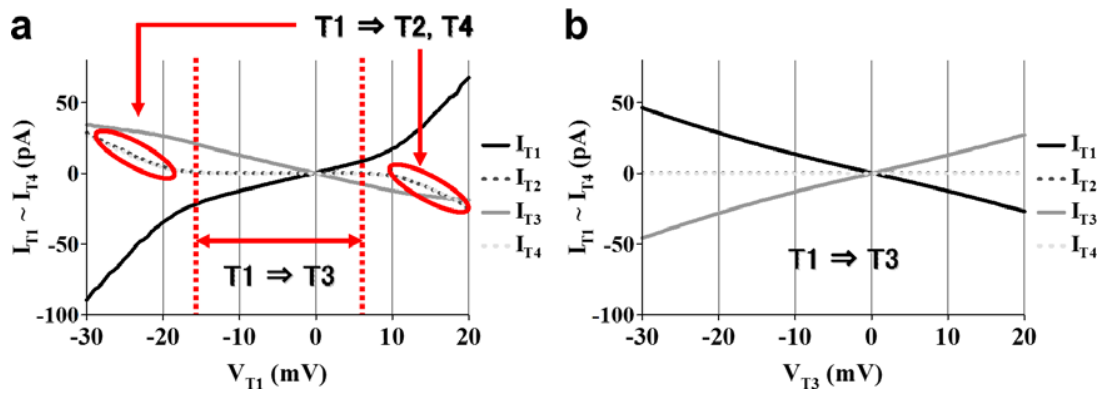

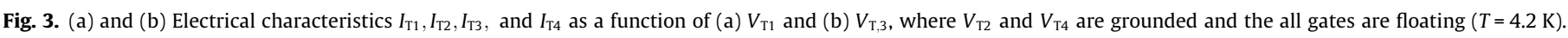

gions of QD1, QD2, and QD3, the bottoms of the conduction band are lower than those at the constrictions, resulting in the formation of the $\mathrm{QDs}$. However, the region of $\mathrm{QD} \alpha$ is just on a constriction. Therefore, the mechanism of quantum confinement for $\mathrm{QD} \alpha$ is different from that of the other QDs. Quantum confinement in $\mathrm{QD} \alpha$ is presumably due to the compressive stress generated during the pattern-dependent oxidation [30] (PADOX) after electron beam lithography. If a connection sandwiched with two large areas is very narrow, compressive stress arises in the connection, lowering the bottom of the conduction band. As a results, $\mathrm{QD} \alpha$ is formed at the constrictions. In contrast to the gate-defined TQDs [21], the very small QD is formed in silicon by the PADOX mechanism. Therefore single-electron tunneling in the TQDs can be observed at a relatively high temperature $(T=4.2 \mathrm{~K})$.

\section{Detailed analysis for electron transport through the silicon serial TQDs}

\subsection{Charge configurations in the charge stability diagram}

To analyze the electron transport through the TQDs in detail, we examine the charge configurations in the charge stability diagram of the TQDs. The charge stability diagram is determined by the electrostatic energy (the calculation is shown in Appendix A) and the quantum-mechanical confinement in the system. Since the quantum level spacings of our QDs (with radii of a few tens of $\mathrm{nm}$ ) are estimated to be a few hundred $\mu \mathrm{eV}$ [31], which is comparable to the thermal energy at the base temperature of $4.2 \mathrm{~K}$ $(\sim 362 \mu \mathrm{eV})$ the quantum-mechanical confinement effects are smeared.

Fig. 4 shows the calculated charge stability diagram from the equivalent circuit simulation, corresponding to the inside of the white square in Fig. 2a. The gray lines mark the boundaries of the charge stable cells in which the stable charge configurations $\left(N_{1}, N_{\alpha}, N_{2}\right)$ are shown, where $N_{1}, N_{\alpha}$, and $N_{2}$ correspond to the charge numbers of $\mathrm{QD} 1, \mathrm{QD} \alpha$, and $\mathrm{QD} 2$, respectively. For simplicity, we assume that $\left(N_{1}, N_{\alpha}, N_{2}\right)=(0,0,0)$ at the hatched region, although there are actually many electrons in the system because of the high doping concentration.

At the boundaries of the charge stable cells, the total electrostatic energies of the neighboring charge states are degenerate. There are three types of boundary in the charge stability diagram of the TQDs. Firstly, since a charging event with a single charge in a QD of the TQDs occurs across, for example, the red lines in 


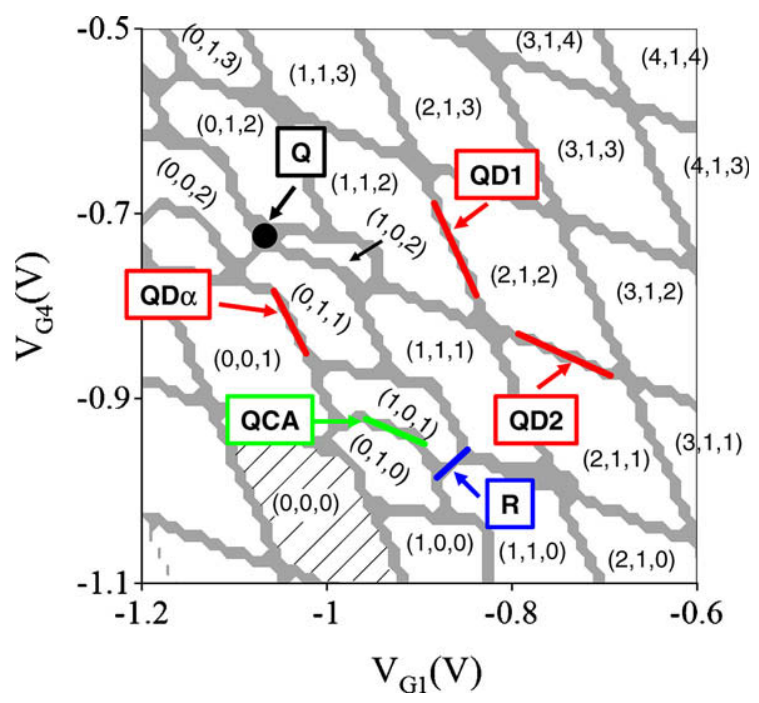

Fig. 4. The simulated charge configurations in the stability diagram of the TQDs. In order to show the sharp boundaries of the charge stable regions, almost zero bias voltages and the temperature of $10 \mathrm{mK}$ are used in the simulation. The stable charge configuration in each cell is denoted by $\left(N_{1}, N_{\alpha}, N_{2}\right)$, where $N_{1}, N_{\alpha}$, and $N_{2}$ correspond to the electron numbers of $\mathrm{QD} 1, \mathrm{QD} \alpha$, and $\mathrm{QD} 2$, respectively. These electron numbers are the relative values from those of the hatched region, where $N_{1}, N_{\alpha}$, and $N_{2}$ are assumed to $(0,0,0)$ for simplicity.

Fig. 4, these lines are referred to as the charging lines. These three red lines correspond to the charging lines of QD1, QD2, and $\mathrm{QD} \alpha$ as indicated in Fig. 4. Many charging lines exist in the stability diagram. Secondly, at the blue line with the mark $\mathrm{R}$ in Fig. 4, a charge at $\mathrm{QD} 2(\alpha)$ moves to $\mathrm{QD} \alpha(2)$, but the total numbers of charges in the TQDs stay constant. This type of line is the charge reconfiguration line, which arises from the electrostatic coupling (and also quantum-mechanical tunnel coupling which is neglected in the simulation). In the charge stability diagram of DQDs, these two types of line also appear [29]. However, the other kind of line indicated by the green line in Fig. 4 is identified in the TQDs, where both a charging event and a charge reconfiguration event occur simultaneously. Since the two charges simultaneously move in the TQDs at this line, in a similar way to quantum cellular automata (QCA) processes [32], this line is referred to as the QCA line [21]. In the experimental results, a strong current peak at the QCA line is observed as indicated by the white arrow in Fig. $2 d$.

\subsection{Charge triple and quadruple points}

The meeting points of two charging lines or a charging line and a QCA line are charge triple points, where three charge configurations are degenerate. Although sequential tunneling is allowed at the charge triple points in the case of the serial DQDs [29], electron transport at the charge triple points in the serial TQDs is a second order tunneling process. However, almost all the current peaks of $I_{\mathrm{T} 1}$ (Fig. 2a) correspond to the charge triple points because our measurement is performed in the non-linear transport region, namely with relatively high bias voltages, and the three electrochemical potentials of QD1, QD2, and QD $\alpha$ located in the bias window. In the region where the current peaks disappear in Fig. 2a, the electrochemical potential of the $\mathrm{QD} \alpha$ is far from the bias window due to the very wide spacing of the charging lines of $\mathrm{QD} \alpha$. This indicates that the charging energy of $\mathrm{QD} \alpha$ is very large (the calculation is discussed below) compared with those of QD1 and QD2, i.e. the size of the $\mathrm{QD} \alpha$ is very small, which is also supported by the SEM observation (Fig. 1a).

Although a two-dimensional charge stability diagram is enough to describe charge configurations in DQDs, a three-dimensional charge stability diagram spanned by three gates is necessary in order to obtain full charge configurations in TQDs, because there are three discrete charges in TQDs. This is referred to as a beehive diagram [21]. In three-dimensional space, charging lines and triple points turn into charging plains and triple lines, respectively, and the meeting points of the two triple lines form charge quadruple points, where the four electron configurations are degenerate. Sequential tunneling in the linear transport region for the serial TQDs is allowed only at the charge quadruple points. At the electron configurations from $(0,0,0)$ to $(1,1,1)$ in a three-dimensional charge stability diagram of the TQDs, there are the four charge quadruple points [21]:

$$
\begin{aligned}
& Q_{A}[(0,0,0) \leftrightarrow(1,0,0) \leftrightarrow(0,1,0) \leftrightarrow(0,0,1)], \\
& Q_{B}[(1,1,1) \leftrightarrow(1,1,0) \leftrightarrow(1,0,1) \leftrightarrow(0,1,1)], \\
& Q_{C}[(1,0,0) \leftrightarrow(0,1,0) \leftrightarrow(1,1,0) \leftrightarrow(1,0,1)], \\
& Q_{D}[(0,0,1) \leftrightarrow(1,0,1) \leftrightarrow(0,1,1) \leftrightarrow(0,1,0)]
\end{aligned}
$$

At $Q_{A}$ and $Q_{B}$, a single charge is transferred through the TQDs, whereas two charges are involved in the transport at $Q_{C}$ and $Q_{D}$. These two charge transport processes can only occur in QD systems comprising more than two QDs.

At the black closed circle indicated by $Q$ in Fig. 4, the charge quadruple point which involves three electrons appears. The four charge configurations $(0,0,2),(1,0,2),(0,1,2)$, and $(0,1,1)$ are degenerate at $Q$ corresponding to $Q_{D}$ with an extra single charge on $\mathrm{QD2}$. Fig. 5a shows the schematic potential diagrams at $\mathrm{Q}$ where the left, center, and right potential wells correspond to those of $\mathrm{QD} 1, \mathrm{QD} \alpha$, and $\mathrm{QD2}$, respectively. The electrochemical potentials $\mu_{1}, \mu_{2}, \mu_{\alpha}, \mu_{\mathrm{T} 1}$, and $\mu_{\mathrm{T} 4}$ are those of QD1, QD2, QD $\alpha$, the terminal $\mathrm{T} 1$, and $\mathrm{T} 4$, respectively. In the $(0,1,1)$ configuration, the electron on $\mathrm{QD} \alpha$ can move to QD2 because $\mu_{\alpha}(0,1,1)=\mu_{2}(0,0,2)$. This transfer occurs below the electrochemical potentials of $\mathrm{T} 1$ and $\mathrm{T} 4$. Then one electron fills
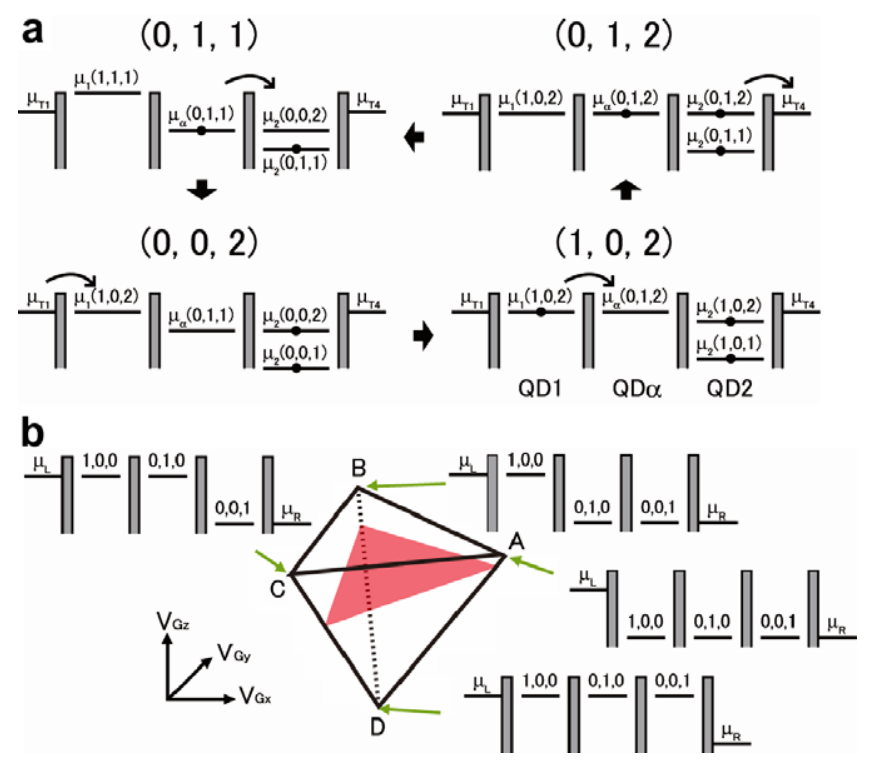

Fig. 5. (a) Schematic diagrams of the electrochemical potentials $\mu_{1}, \mu_{\alpha}$, and $\mu_{2}$ of the $\mathrm{QD} 1, \mathrm{QD} \alpha$, and $\mathrm{QD} 2$, respectively, at the charge quadruple point $\mathrm{Q}$ in Fig. 4 $\mu_{\mathrm{T} 1}$ and $\mu_{\mathrm{T} 4}$ are the electrochemical potentials at $\mathrm{T} 1$ and $\mathrm{T} 4$, respectively. The values inside the parentheses next to the electrochemical potentials indicate the electron configurations of $\mathrm{QD} 1, \mathrm{QD} \alpha$, and QD2. (b) Schematic diagram of the non-linear transport at the charge quadruple point $Q_{A}$. The electrochemical potentials of charge configurations $(1,0,0),(0,1,0)$, and $(0,0,1)$ are illustrated in the potential diagrams. $\mu_{L}$ and $\mu_{R}$ are the electrochemical potential of the leads. $V_{\mathrm{Gx}}, V_{\mathrm{Gy}}$, and $V_{\mathrm{Gz}}$ are three gate voltages. The red triangle indicates the twodimensional slice of the triangular pyramid in the $V_{\mathrm{Gx}}-V_{\mathrm{Gy}}$ plain. 
$\mu_{1}(1,0,2)$ from $\mathrm{T} 1$, resulting in the $(1,0,2)$ configuration. The configuration of $\mu_{1}(1,0,2)=\mu_{\alpha}(0,1,2)$ allows the tunneling of the electron from $\mathrm{QD} 1$ to $\mathrm{QD} \alpha$. Finally, the electron on $\mathrm{QD} 2$ escapes to $\mathrm{T} 4$ and the configuration returns to the initial state $(0,1,1)$. Note that the sequential tunneling at $\mathrm{Q}$ is different from that at $Q_{A}$ and $Q_{B}$ because of the different number of charges. In addition, spin blockade between $\mathrm{QD} \alpha$ and QD2 is expected to appear in this charge configuration because the two specific conditions $(0,1,1)$ and $(0,0,2)$ are involved, where the two electron spins can form the spin singlet or triplet. The study of spin blockade in TQDs remains as a matter to be discussed further.

\subsection{Charging energies of the TQDs}

From the parameters used for the equivalent circuit simulation, the charging energies of $\mathrm{QD} 1, \mathrm{QD} 2$, and $\mathrm{QD} \alpha\left(E_{\mathrm{QD} 1}, E_{\mathrm{QD} 2}\right.$, and $\left.E_{\mathrm{QD} \alpha}\right)$, and the electrostatic coupling energies between QD1(2) and QD $\alpha$ $\left[E_{1(2) \alpha}\right]$ are calculated from the electrostatic energies of the system (a full derivation is shown in Appendix A). These energies obtained are $E_{\mathrm{QD} 1} \sim 5.6 \mathrm{meV}, \quad E_{\mathrm{QD} 2} \sim 10.4 \mathrm{meV}, \quad E_{\mathrm{QD} \alpha} \sim 101 \mathrm{meV}, \quad E_{1 \alpha} \sim$ $3.6 \mathrm{meV}$, and $E_{2 \alpha} \sim 3.3 \mathrm{meV}$. Note that $E_{\mathrm{QD} \alpha}$ is very large, resulting in the very wide spacing of the charging lines of $\mathrm{QD} \alpha$, corresponding to a very small size for $\mathrm{QD} \alpha$ ( $\sim$ a few nm radius). The PADOX process in the silicon nanostructures allows us to realize such a small QD.

In contrast, for the estimation of the charging energies from the experimental results, the conversion factors by which gate voltages are converted into energies, must be extracted from the non-linear transport results [29]. Fig. 5b shows the schematic image of the non-linear transport of the charge quadruple point $Q_{A}$ in the three-dimensional stability diagram spanned by the three gate voltages $V_{\mathrm{Gx}}, V_{\mathrm{Gy}}$, and $V_{\mathrm{Gz}}$ for TQDs. In non-linear transport, the charge quadruple point turns into the triangular pyramid (the black bold and dotted lines). On the $V_{\mathrm{Gx}}-V_{\mathrm{Gy}}$ plain, a two-dimensional slice appears as illustrated by the red triangle in Fig. 5. The configurations of the electrochemical potentials at the four vertices $A, B, C$, and $D$ are also shown. The line $A D$ corresponds to the charge reconfiguration line for the three QDs. To extract the accurate conversion factors for three QDs, the two side of the triangular pyramid must be identified.

In the experimental results (Fig. 2a), several triangular current peaks appear, corresponding to just the two-dimensional slice of the triangular pyramids. However, the charging energies can be roughly estimated from the triangles in two-dimensional space. Since G1(G4) is strongly coupled to QD1(QD2), we obtain the conversion factors of the QD1 and $\operatorname{QD} 2\left(\gamma_{1}\right.$ and $\left.\gamma_{2}\right)$ from the triangle of the $V_{\mathrm{G} 1}-V_{\mathrm{G} 4}$ plain. We chose the clearest triangle indicated by the white arrow in Fig. 2a. If we assume that the bias voltages drop only at the tunnel junctions, $\gamma_{1(2)}$ is calculated as 0.061 $(0.071) \mathrm{eV} / \mathrm{V}$. From the conversion factor and the current peak spacing of QD1(2), the charging energy of QD1(2) is estimated as 7.7 (10.3) meV. These values are consistent with the charging energies calculated from the equivalent circuit parameters, probably because the triangle shape is close to the side of the triangular pyramid due to the small capacitive couplings between $\mathrm{QD} \alpha$ and $\mathrm{G} 1$ or G4.

\subsection{Higher order tunneling processes}

Finally, we explore the higher order tunneling processes of the TQDs. In the transport of the TQDs, several kinds of tunneling processes appear. The charge quadruple points and the charge triple points correspond to first and second order tunneling processes, respectively. The leakage currents of the Coulomb blockade region are due to fourth order tunneling processes. On the QCA lines (the green line in Fig. 4), where two charges move simultaneously, an-
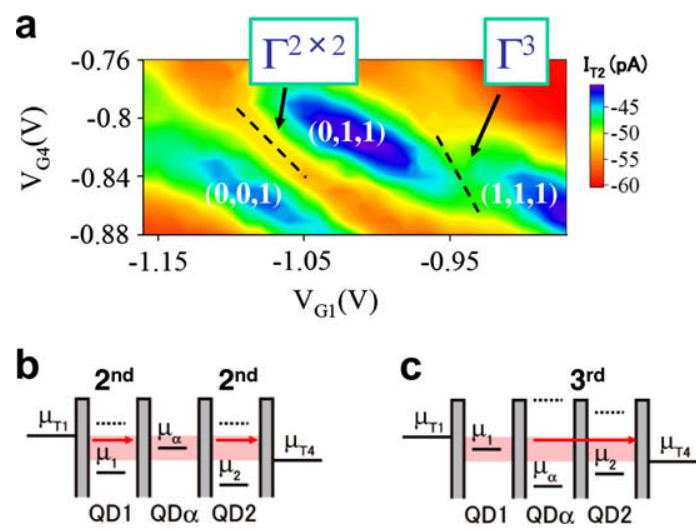

Fig. 6. (a) The extended figure corresponding to the blue square as shown in Fig. 2b. The electron configurations extracted from the calculation (Fig. 4). (b) and (c) Schematic potential diagrams of the TQDs at the left and the right black broken line in Fig. 6a.

other higher order tunneling process occurs, which is referred to as QCA cotunneling processes [21].

Here, we compare the other two kinds of higher order tunneling processes. Fig. 6a shows the extended figure corresponding to the blue square in Fig. 2b, where the electron configurations assumed in Fig. 4 are denoted. In this gate voltage region, the three electron configurations $(0,0,1),(0,1,1)$, and $(1,1,1)$ are distinguished. Between $(0,0,1)$ and $(0,1,1)$, only the electrochemical potential of $\mathrm{QD} \alpha$ locates in the bias window. As a result, two successive second order tunneling processes with a tunneling rate of $\Gamma^{2 \times 2}$ are required in the electron transport as schematically shown in Fig. $6 \mathrm{~b}$. Conversely, at the boundary of $(0,1,1)$, and $(1,1,1)$, there is only the electrochemical potential of QD1 in the bias window and the electron transport is dominated by the third order tunneling processes with a tunneling rate of $\Gamma^{3}$ shown in Fig. 6c. The black broken lines in Fig. 6a indicate these two tunneling processes. Note that the tunneling rate of the two successive second order tunneling process is higher than that of the third order processes, leading to the different current amplitudes in Fig. 6a. This fact is consistent with other TQD systems $[21,24]$.

\section{Conclusion}

Experimental electron transport through the silicon serial TQD system has been successfully observed for the first time at a temperature of $4.2 \mathrm{~K}$. The TQD system is composed of two lithographically-patterned QDs and another one in-between formed by compressive stress generated during the PADOX process [30]. The equivalent circuit simulation shows an excellent agreement with the experimental results, which support the assumed formation of the TQDs. The calculated charge configurations of the TQDs, which are defined by three types of lines (the charging lines, the charge reconfiguration lines, and the QCA lines), provide a detailed analysis of the electron transport for the TQD system, including a discussion of the charge quadruple point which involves three electrons. We also discussed the charge quadruple point at the non-linear transport region for the estimation of the charging energies. From the experimental data, we can roughly estimate the charging energies, which are consistent with the calculated values from the simulation. In addition, we discussed the several kinds of higher order tunneling processes, including the comparison between the two successive second order tunneling processes and the third order tunneling processes. The TQD system provides an essential prerequisite for the future implementation of siliconbased quantum information processing devices because of its high functionality. 


\section{Acknowledgements}

We thank Tasuku Nagami and Stuart Boden for valuable discussions. This work was partly supported by KAKENHI (19.10625). One of authors, (G.Y.), is supported by the JSPS Research Fellowship for Young Scientists.

\section{Appendix A. Charging energies for the multiple QDs}

The charging energies for the multiple QDs can be derived from the electrostatic energy of the system [29]. In a $N$ conductors system, the node $j$ is connected to the other nodes with the capacitances $c_{j k}$ and the stored charge $Q_{j}$ is given by:

$Q_{j}=\sum_{k} c_{j k}\left(V_{j}-V_{k}\right)$.

where $V_{j}$ is the electrostatic potential of the node $j$. This equation can be expressed in the matrix form

$\vec{Q}=\boldsymbol{C} \vec{V}$.

Here $\boldsymbol{C}$ is the capacitance matrix, which consists of the diagonal matrix elements $C_{j j}=\sum_{k \neq j} c_{j k}$ and the off-diagonal matrix elements $C_{j k}=C_{k j}=-C_{j k}$. To consider the voltage sources in the network, the matrix equation is separated as

$$
\left(\begin{array}{l}
\vec{Q}_{N} \\
\vec{Q}_{S}
\end{array}\right)=\left(\begin{array}{ll}
\boldsymbol{C}_{N N} & \boldsymbol{c}_{N S} \\
\boldsymbol{C}_{S N} & \boldsymbol{C}_{S S}
\end{array}\right)\left(\begin{array}{l}
\vec{V}_{N} \\
\vec{V}_{S}
\end{array}\right),
$$

where $\vec{Q}_{N(S)}$ and $\vec{V}_{N(S)}$ are the charges and the voltages on the charge nodes (the voltage sources), respectively, and $\boldsymbol{C}_{N N}, \boldsymbol{C}_{N S}, \boldsymbol{C}_{S N}$, and $\boldsymbol{C}_{S S}$ are the four capacitance sub-matrices derived from $\boldsymbol{C}$. In this expression, the voltage sources are treated as nodes with large capacitances to the ground. Although many voltage sources are set in our systems, it is not necessary to obtain $\boldsymbol{C}_{S N}$ and $\boldsymbol{C}_{S S}$ because the values of the voltage sources are already known. Therefore, from the voltages on the charge nodes

$\vec{V}_{N}=\boldsymbol{C}_{N N}^{-1}\left(\vec{Q}_{N}-\boldsymbol{C}_{N S} \vec{V}_{S}\right)$,

the electrostatic energy of the charge nodes can be determined as follows:

$U=\frac{1}{2} \vec{V}_{N} \cdot \boldsymbol{C}_{N N} \vec{V}_{N}$

From the equivalent circuit for our multiple QDs (Fig. 1b), the charges on $\mathrm{QDi}(i=1,2,3, \alpha)$, which are expressed such that $Q_{i}=-N_{i}|e|$, where $N_{i}$ is the number of electrons on QDi and $-|e|$ is the electron charge, are given by

$Q_{1}=C_{1}\left(V_{1}-V_{\mathrm{T} 1}\right)+C_{2}\left(V_{1}-V_{3}\right)+C_{6}\left(V_{1}-V_{\alpha}\right)$

$+C_{11}\left(V_{1}-V_{\mathrm{G} 1}\right)+C_{41}\left(V_{1}-V_{\mathrm{G} 4}\right)+C_{1 p} V_{1}$,

$Q_{2}=C_{4}\left(V_{2}-V_{\mathrm{T} 2}\right)+C_{5}\left(V_{2}-V_{\mathrm{T} 4}\right)+C_{7}\left(V_{2}-V_{\alpha}\right)$

$+C_{12}\left(V_{2}-V_{\mathrm{G} 1}\right)+C_{42}\left(V_{2}-V_{\mathrm{G} 4}\right)+C_{23}\left(V_{2}-V_{3}\right)+C_{2 p} V_{2}$,

$Q_{3}=C_{2}\left(V_{3}-V_{1}\right)+C_{3}\left(V_{3}-V_{\mathrm{T} 3}\right)+C_{13}\left(V_{3}-V_{\mathrm{G} 1}\right)$ $+C_{\alpha 3}\left(V_{3}-V_{\alpha}\right)+C_{23}\left(V_{3}-V_{2}\right)+C_{3 p} V_{3}$

$Q_{\alpha}=C_{6}\left(V_{\alpha}-V_{1}\right)+C_{7}\left(V_{\alpha}-V_{2}\right)+C_{1 \alpha}\left(V_{\alpha}-V_{\mathrm{G} 1}\right)$

$$
+C_{4 \alpha}\left(V_{\alpha}-V_{\mathrm{G} 4}\right)+C_{\alpha 3}\left(V_{\alpha}-V_{3}\right) \text {. }
$$

These equations can be written in the matrix form as

$$
\begin{gathered}
\left(\begin{array}{l}
Q_{1}+C_{1} V_{\mathrm{T} 1}+C_{11} V_{\mathrm{G} 1}+C_{41} V_{\mathrm{G} 4} \\
Q_{2}+C_{4} V_{\mathrm{T} 2}+C_{5} V_{\mathrm{T} 4}+C_{12} V_{\mathrm{G} 1}+C_{42} V_{\mathrm{G} 4} \\
Q_{3}+C_{3} V_{\mathrm{T} 3}+C_{13} V_{\mathrm{G} 1} \\
Q_{\alpha}+C_{1 \alpha} V_{\mathrm{G} 1}+C_{4 \alpha} V_{\mathrm{G} 4}
\end{array}\right) \\
=\left(\begin{array}{cccc}
C_{\mathrm{QD} 1} & 0 & -C_{2} & -C_{6} \\
0 & C_{Q D 2} & -C_{23} & -C_{7} \\
-C_{2} & -C_{23} & C_{Q D 3} & -C_{\alpha 3} \\
-C_{6} & -C_{7} & -C_{\alpha 3} & C_{Q D \alpha}
\end{array}\right)\left(\begin{array}{l}
V_{1} \\
V_{2} \\
V_{3} \\
V_{\alpha}
\end{array}\right),
\end{gathered}
$$

where $C_{Q D 1}=C_{1}+C_{2}+C_{6}+C_{11}+C_{41}+C_{1 p}, \quad C_{Q D 2}=C_{4}+C_{5}+C_{7}+$ $C_{42}+C_{12}+C_{23}+C_{2 p}, \quad C_{Q D 3}=C_{2}+C_{3}+C_{13}+C_{23}+C_{\alpha 3}+C_{3 p}, \quad$ and $C_{Q D \alpha}=C_{6}+C_{7}+C_{1 \alpha}+C_{4 \alpha}+C_{\alpha 3}$. From the matrix equation Eq. (10), we obtain $\vec{V}_{N}$. As a result, the electrostatic energy $U\left(N_{1}, N_{2}, N_{3}, N_{\alpha}\right)$ can be calculated with Eq. (5).

The electrochemical potentials $\mu_{i}$, which are defined as the energy required to add the $N_{i}$ th electron to QDi, are given by

$\mu_{1}\left(N_{1}, N_{2}, N_{3}, N_{\alpha}\right)=U\left(N_{1}, N_{2}, N_{3}, N_{\alpha}\right)-U\left(N_{1}-1, N_{2}, N_{3}, N_{\alpha}\right)$,

$\mu_{2}\left(N_{1}, N_{2}, N_{3}, N_{\alpha}\right)=U\left(N_{1}, N_{2}, N_{3}, N_{\alpha}\right)-U\left(N_{1}, N_{2}-1, N_{3}, N_{\alpha}\right)$,

$\mu_{3}\left(N_{1}, N_{2}, N_{3}, N_{\alpha}\right)=U\left(N_{1}, N_{2}, N_{3}, N_{\alpha}\right)-U\left(N_{1}, N_{2}, N_{3}-1, N_{\alpha}\right)$,

$\mu_{\alpha}\left(N_{1}, N_{2}, N_{3}, N_{\alpha}\right)=U\left(N_{1}, N_{2}, N_{3}, N_{\alpha}\right)-U\left(N_{1}, N_{2}, N_{3}, N_{\alpha}-1\right)$.

The charging energies (the addition energies) for the QD are

$E_{Q D 1}=\mu_{1}\left(N_{1}+1, N_{2}, N_{3}, N_{\alpha}\right)-\mu_{1}\left(N_{1}, N_{2}, N_{3}, N_{\alpha}\right)$,

$E_{Q D 2}=\mu_{2}\left(N_{1}, N_{2}+1, N_{3}, N_{\alpha}\right)-\mu_{2}\left(N_{1}, N_{2}, N_{3}, N_{\alpha}\right)$,

$E_{Q D 3}=\mu_{3}\left(N_{1}, N_{2}, N_{3}+1, N_{\alpha}\right)-\mu_{3}\left(N_{1}, N_{2}, N_{3}, N_{\alpha}\right)$,

$E_{Q D \alpha}=\mu_{\alpha}\left(N_{1}, N_{2}, N_{3}, N_{\alpha}+1\right)-\mu_{\alpha}\left(N_{1}, N_{2}, N_{3}, N_{\alpha}\right)$,

giving

$$
\begin{aligned}
E_{Q D 1} & =|e|^{2} \beta\left(2 C_{23} C_{7} C_{\alpha 3}+C_{\alpha 3}^{2} C_{Q D 2}+C_{23}^{2} C_{Q D \alpha}\right. \\
& \left.+C_{Q D 3}\left(C_{7}^{2}-C_{Q D 2} C_{Q D \alpha}\right)\right), \\
E_{Q D 2} & =|e|^{2} \beta\left(2 C_{2} C_{6} C_{\alpha 3}+C_{\alpha 3}^{2} C_{Q D 1}+C_{2}^{2} C_{Q D \alpha}\right. \\
& \left.+C_{Q D 3}\left(C_{6}^{2}-C_{Q D 1} C_{Q D \alpha}\right)\right), \\
E_{Q D 3} & =|e|^{2} \beta\left(C_{7}^{2} C_{Q D 1}+C_{Q D 2}\left(C_{6}^{2}-C_{Q D 1} C_{Q D \alpha}\right)\right), \\
E_{Q D \alpha} & =|e|^{2} \beta\left(C_{23}^{2} C_{Q D 1}+C_{Q D 2}\left(C_{2}^{2}-C_{Q D 1} C_{Q D 3}\right)\right),
\end{aligned}
$$

where

$$
\begin{aligned}
1 / \beta= & 2 C_{23} C_{7}\left(C_{2} C_{6}+C_{\alpha 3} C_{Q D 1}\right)+2 C_{2} C_{6} C_{\alpha 3} C_{Q D 2}+C_{\alpha 3}^{2} C_{Q D 1} C_{Q D 2} \\
& +C_{7}^{2} C_{Q D 1} C_{Q D 3}+C_{6}^{2} C_{Q D 2} C_{Q D 3}-C_{Q D 1} C_{Q D 2} C_{Q D 3} C_{Q D \alpha} \\
& +C_{23}^{2}\left(-C_{6}^{2}+C_{Q D 1} C_{Q D \alpha}\right)+C_{2}^{2}\left(-C_{7}^{2}+C_{Q D 2} C_{Q D \alpha}\right) .
\end{aligned}
$$

In addition, the electrostatic coupling energies between QD1(2) and $\mathrm{QD} \alpha\left[E_{1(2) \alpha}\right]$ are determined by

$$
\begin{aligned}
& E_{1 \alpha}=\mu_{1}\left(N_{1}, N_{2}, N_{3}, N_{\alpha}+1\right)-\mu_{1}\left(N_{1}, N_{2}, N_{3}, N_{\alpha}\right) \\
& =|e|^{2} \beta\left(C_{23}^{2} C_{6}-C_{2} C_{23} C_{7}-C_{2} C_{\alpha 3} C_{Q D 2}-C_{6} C_{Q D 2} C_{Q D 3}\right) \text {, } \\
& E_{2 \alpha}=\mu_{2}\left(N_{1}, N_{2}, N_{3}, N_{\alpha}+1\right)-\mu_{2}\left(N_{1}, N_{2}, N_{3}, N_{\alpha}\right) \\
& =|e|^{2} \beta\left(C_{2}^{2} C_{7}-C_{2} C_{23} C_{6}-C_{23} C_{\alpha 3} C_{Q D 1}-C_{7} C_{Q D 1} C_{Q D 3}\right) \text {. }
\end{aligned}
$$

\section{References}

[1] Hayashi T, Fujisawa T, Cheong HD, Jeong YH, Hirayama Y. Coherent manipulation of electronic states in a double quantum dot. Phys Rev Lett 2003;91:226804.

[2] Petta JR, Johnson AC, Taylor JM, Laird EA, Yacoby A, Lukin MD, et al. Coherent manipulation of coupled electron spins in semiconductor quantum dots. Science 2005;309:2180.

[3] Koppens FHL, Buizert C, Tielrooij KJ, Vink IT, Nowack KC, Meunier T, et al Driven coherent oscillations of a single electron spin in a quantum dot. Nature 2006;442:766.

[4] Johnson AC, Petta JR, Taylor JM, Yacoby A, Lukin MD, Marcus CM, et al. Tripletsinglet spin relaxation via nuclei in a double quantum dot. Nature 2005;435:925.

[5] Goswami S, Slinker KA, Friesen M, McGuire LM, Truitt JL, Tahan C, et al. Controllable valley splitting in silicon quantum devices. Nat Phys 2007;3:41.

[6] Fujiwara A, Inokawa H, Yamazaki K, Namatsu H, Takahashi Y, Zimmerman NM, et al. Single electron tunneling transistor with tunable barriers using silicon nanowire metal-oxide-semiconductor field-effect transistor. Appl Phys Lett 2006;88:053121.

[7] Liu H, Fujisawa T, Inokawa H, Ono Y, Fujiwara A, Hirayama Y. A gate-defined silicon quantum dot molecule. Appl Phys Lett 2008;92:222104.

[8] Chan VC, Buehler TM, Ferguson AJ, McCamey DR, Reilly DJ, Dzurak AS, et al. Ion implanted Si:P double dot with gate tunable interdot coupling. J Appl Phys 2006;100:106104. 
[9] Hu Y, Churchill HOH, Reilly DJ, Xiang J, Lieber CM, Marcus CM. A Ge/S heterostructure nanowire-based double quantum dot with integrated charge sensor. Nat Nanotechnol 2007;2:622.

[10] Ono K, Austing DG, Tokura Y, Tarucha S. Current rectification by Paul exclusion in a weakly coupled double quantum dot system. Science 2002;297:1313.

[11] Johnson AC, Petta JR, Marcus CM, Hanson MP, Gossard AC. Singlet-triplet spin blockade and charge sensing in a few-electron double quantum dot. Phys Rev B 2005;72:165308.

[12] Shaji N, Simmons CB, Thalakulam M, Klein LJ, Qin H, Luo H, et al. Spin blockade and lifetime-enhanced transport in a few-electron $\mathrm{Si} / \mathrm{SiGe}$ double quantum dot. Nat Phys 2008;4:540.

[13] Liu HW, Fujisawa T, Ono Y, Inokawa H, Fujiwara A, Takashina K, et al. Paulispin-blockade transport through a silicon double quantum dot. Phys Rev B 2008;77:073310.

[14] Hada Y, Eto M. Exchange coupling in silicon double quantum dots. Jpn J Appl Phys 2004;43:7329.

[15] DiVincenzo DP, Bacon D, Kempe J, Burkard G, Whaley KB. Universal quantum computation with the exchange interaction. Nature 2000;408: 339.

[16] Hawrylak P, Korkusinski M. Voltage-controlled coded qubit based on electron spin. Solid State Commun 2005;136:508.

[17] Meier F, Levy J, Loss D. Quantum computing with spin cluster qubits. Phys Rev Lett 2003;90:047901.

[18] Saraga DS, Loss D. Spin-entangled currents created by a triple quantum dot Phys Rev Lett 2003;90:166803.

[19] Stopa M. Rectifying behavior in Coulomb blockades: charging rectifiers. Phys Rev Lett 2002;88:146802.

[20] Vidan A, Westervelt RM, Stopa M, Hanson M, Gossard AC. Triple quantum dot charging rectifier. Appl Phys Lett 2004;85:3602.
[21] Schröer D, Greentree AD, Gaudreau L, Eberl K, Hollenberg LCL, Kotthaus JP, et al. Electrostatically defined serial triple quantum dot charged with few electrons. Phys Rev B 2007;76:075306.

[22] Gaudreau L, Studenikin SA, Sachrajda AS, Zawadzki P, Kam A, Lapointe J, et al. Stability diagram of a few-electron triple dot. Phys Rev Lett 2006;97:036807.

[23] Rogge MC, Haug RJ. Two-path transport measurements on a triple quantum dot. Phys Rev B 2008;77:193306.

[24] Grove-Rasmussen K, Jørgensen HI, Hayashi T, Lindelof PE, Fujisawa T. A triple quantum dot in a single-wall carbon nanotube. Nano Lett 2008;8:1055.

[25] Amaha S, Hatano T, Teraoka S, Shibatomi A, Tarucha S, Nakata Y, et al. Laterally coupled self-assembled InAs quantum dots embedded in resonant tunnel diode with multigate electrodes. Appl Phys Lett 2008;92:202109.

[26] Single C, Prins FE, Kern DP. Simultaneous operation of two adjacent double dots in silicon. Appl Phys Lett 2001;78:1421.

[27] Macucci M, Gattobigio M, Bonci L, Iannaccone G, Prins FE, Single C, et al. A QCA cell in silicon-on-insulator technology: theory and experiment. Superlattices Microstruct 2003;34:205

[28] Khalafalla MAH, Mizuta H, Durrani ZAK. Switching of single-electron oscillations in dual-gated nanocrystalline silicon point-contact transistors. IEEE Trans Nanotechnol 2003;2(4):271.

[29] van der Wiel WG, Franceschi SD, Elzerman JM, Fujisawa T, Tarucha S, Kouwenhoven LP. Electron transport through double quantum dots. Rev Mod Phys 2003;75:1.

[30] Horiguchi S, Nagase M, Shiraishi K, Kageshima H, Takahashi Y, Murase K. Mechanism of potential profile formation in silicon single-electron transistors fabricated using patter-dependent oxidation. Jpn J Appl Phys 2001;40:L29.

[31] Angus SJ, Ferguson AJ, Dzurak AS, Clark RG. gate-defined quantum dots in intrinsic silicon. Nano Lett 2007;7:2051.

[32] Lent CS, Tougaw PD, Porod W, Bernstein GH. Quantum cellular automata. Nanotechnology 1993;4:49. 